\title{
Posterior Reversible Encephalopathy Syndrome in a Critically III Postoperative Patient
}

\author{
Min Ae Keum, M.D., Hyo Keun No, M.D., Choong Wook Lee, M.D., , Sang-Beom Jeon, M.D. , \\ and Suk-Kyung Hong, M.D., Ph.D.
}

Division of Trauma and Surgical Critical Care, Department of Surgery, University of Ulsan College of Medicine, Asan Medical Center, *Department of Radiology and Research Institute of Radiology, Asan Medical Center, University of Ulsan College of Medicine, `Department of Neurology, Asan Medical Center, University of Ulsan College of Medicine, Seoul, Korea

Posterior reversible encephalopathy syndrome (PRES) is a transient condition characterized by altered mental status, seizure, headache, and visual disturbance with typical neuro-imaging findings in the bilateral parieto-occipital regions. Clinicians should be aware of this syndrome because delayed diagnosis and treatment result in irreversible neurologic deficits. We present the case of a 77-yearold male diagnosed with PRES in the setting of postoperative critical illness caused by small-bowel strangulation.

Key Words: intensive care units; posterior leukoencephalopathy syndrome; postoperative period.

Posterior reversible encephalopathy syndrome (PRES) was first described as reversible posterior leukoencephalopathy syndrome by Hinchey et al.[1] in 1996. The primary symptoms included headache, vomiting, altered mental status, seizures, and visual disturbance[1,2] in the presence with typical, reversible, neuro-imaging findings of vasogenic edema involving the subcortical white matter in the posterior portion of the cerebral hemisphere, especially in the bilateral parieto-occipital regions.[2-4] Various clinical conditions were identified as presenting a risk for PRES, such as abrupt arterial hypertension, impaired renal function, eclampsia/pre-eclampsia, an immunosuppressive/cytotoxic agent, transplantation, autoimmune diseases, infection, etc.[5] The pathophysiology of PRES remains controversial, although the favored theory is failure of cerebral auto-regulation due to an abrupt rise in blood pressure resulting in blood-brain barrier disruption with reversible vasogenic edema.[1,3,5] The incidence of PRES is unknown, although its recognition and diagnosis are increasing. Although PRES remains unfamiliar to many clinicians, it is important to be aware of this syndrome as early diagnosis and treatment can lead to reversible results and a better prognosis. We report the case of a patient diagnosed with PRES in the setting of a postoperative critical illness following emergent abdominal surgery and intra-operative cardiopulmonary resuscitation (CPR) due to small-bowel strangulation.

\section{Case Report}

Received on September 24, 2014 Revised on November 19, 2014 Accepted on December 3, 2014

Correspondence to: Suk-Kyung Hong, Division of Trauma and Surgical Critical Care, Department of Surgery, University of Ulsan College of Medicine, Asan Medical Center, 88, Olympic-ro 43-gil, Songpa-gu, Seoul 138-736, Korea Tel: +82-2-3010-3510, Fax: +82-2-3010-6701

E-mail: skhong94@amc.seoul.kr

*No potential conflict of interest relevant to this article was reported.
A 77-year-old man was transferred to the emergency department with a five-day history of abdominal pain, gradually progressive vomiting, watery diarrhea, and dyspnea despite conservative treatment for paralytic ileus at a local hospital. The patient had a history of subtotal gastrectomy and adju- 
Table 1. Laboratory data

\begin{tabular}{|c|c|c|}
\hline Variable & Reference range & On admission to emergency department \\
\hline Hemoglobin (g/dL) & 13-17 & 11.8 \\
\hline White-cell count (per mm³) & $4,000-10,000$ & 2,400 \\
\hline \multicolumn{3}{|l|}{ Differential count (\%) } \\
\hline Neutrophils & $50-75$ & 79 \\
\hline Lymphocytes & $20-44$ & 13.4 \\
\hline Monocytes & $2-9$ & 3.4 \\
\hline Platelet count (per mm³) & $150,000-350,000$ & 93,000 \\
\hline Prothrombin time (sec) & $70-140$ & 47.8 \\
\hline $\mathrm{aPTT}(\mathrm{sec})$ & $25-35$ & 36.9 \\
\hline Glucose (mg/dL) & $70-99$ & 116 \\
\hline Creatinine (mg/dL) & $0.70-1.40$ & 2.20 \\
\hline BUN (mg/dL) & $10-26$ & 43 \\
\hline Total protein (g/dL) & $6-8$ & 4.5 \\
\hline Albumin (g/dL) & $3.5-5.2$ & 2.3 \\
\hline AST (IU/L) & $\sim 40$ & 88 \\
\hline ALT (IU/L) & $\sim 40$ & 23 \\
\hline Total bilirubin (mg/dL) & $0.2-1.2$ & 0.2 \\
\hline C-reactive protein (mg/dL) & $0-0.6$ & 20.09 \\
\hline Procalcitonin (ng/mL) & $\sim 0.5$ & 154.26 \\
\hline
\end{tabular}

aPTT: activated partial thromboplastin time; BUN: blood urea nitrogen; AST: aspartate aminotransfertase; ALT: alanine aminotransferase.

vant chemotherapy for advanced gastric cancer performed in 1997, and had already been taking oral hypoglycemic agents for diabetes mellitus for six years. Due to his chronic kidney disease, he had been regularly seen in the outpatient Department of Nephrology, although without dialysis for six months.

On examination, he was alert and his vital signs were 96/62 $\mathrm{mmHg}, 102$ beats/min, 30 breaths/min, and $36.2^{\circ} \mathrm{C}$. His abdomen was rigid and severely distended with decreased bowel sound, tenderness, and rebound tenderness in the whole abdomen. The initial laboratory findings were presented in Table 1. Arterial blood gas analysis revealed a $\mathrm{pH}$ of 7.26, $\mathrm{PaCO}_{2} 18 \mathrm{mmHg}, \mathrm{PaO}_{2} 79 \mathrm{mmHg}, \mathrm{HCO}_{3} 8.1$ $\mathrm{mmol} / \mathrm{L}$, and $\mathrm{SaO}_{2} 93 \%$ while on room air. His lactate level was $4.6 \mathrm{mmol} / \mathrm{L}$.

An abdominal X-ray showed no bowel gas pattern, and abdominal computed tomography scan showed diffuse distension of his small bowel with abrupt luminal narrowing at the ileum. Because the patient was diagnosed clinically as panperitonitis with small bowel strangulation, emergent surgery was performed. The operative finding was closed loop obstruction with small-bowel strangulation caused by an adhesive band. Adhesiolysis, small-bowel resection, and anastomosis were then performed. Immediately after anesthesia induction and endotracheal intubation, cardiac arrest occurred, and one cycle of cardiopulmonary resuscitation was then performed with epinephrine injection. After recovery of his spontaneous circulation, his systolic blood pressure abruptly increased to $200 \mathrm{mmHg}$ and sustained for $5 \mathrm{~min}$ utes. In order to normalize his blood pressure, nicardipine was administered and then systolic blood pressure decreased to $130 \mathrm{mmHg}$ in 10 minutes. Five minutes later, blood pressure decreased under $100 \mathrm{mmHg}$, a continuous infusion of $0.1 \mu \mathrm{g} / \mathrm{kg} / \mathrm{min}$ epinephrine was maintained during the surgery to keep the systolic blood pressure between 120 and $140 \mathrm{mmHg}$. Six hours later after intensive care unit (ICU) administration, systolic blood pressure decreased abruptly to $50 \mathrm{mmHg}$ and portable echocardiography showed diminished myocardial contractility. So continuous infusion of 10 $\mu \mathrm{g} / \mathrm{kg} / \mathrm{min}$ dobutamine was started and then systolic blood pressure was maintained above $100 \mathrm{mmHg}$. All vasopressors were discontinued on postoperative day three. When 

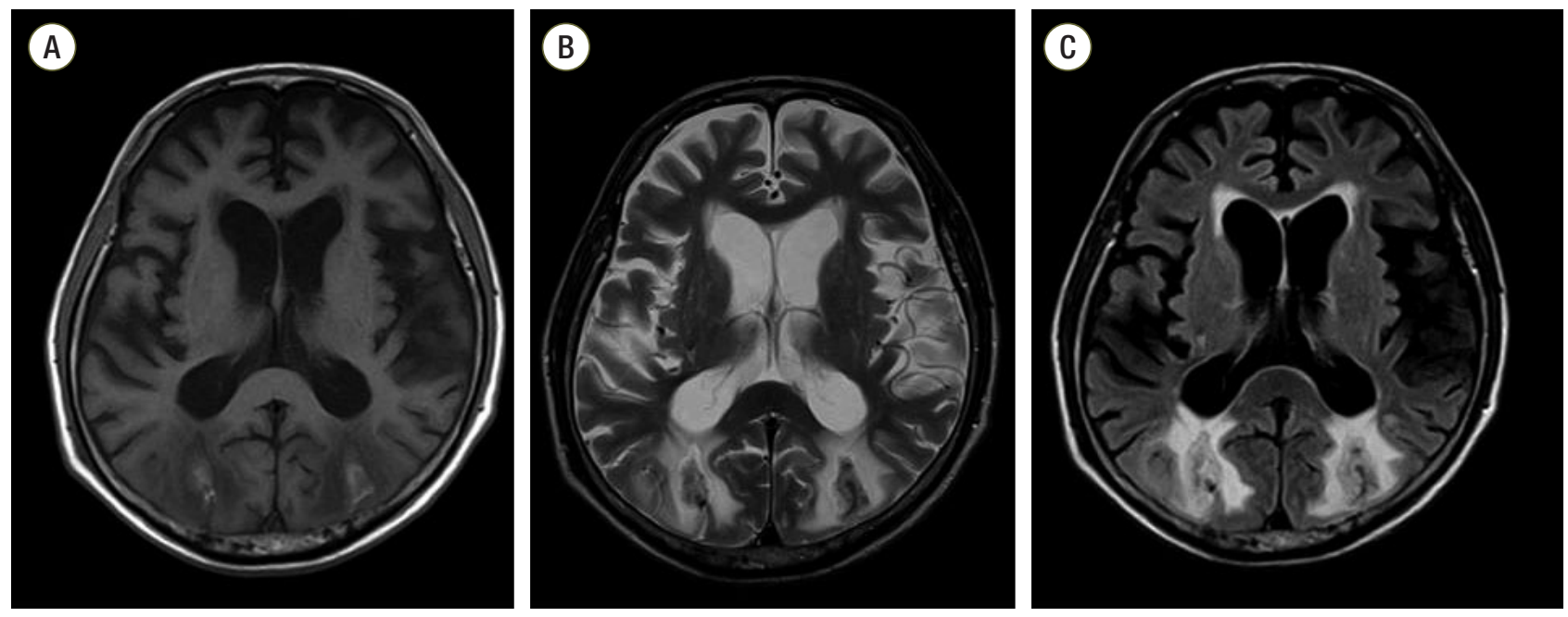

Fig. 1. MRI assessment of chronic changes related to PRES with internal intracerebral hemorrhage. Axial T1-weighted imaging (A) and axial T2-weighted imaging (B) revealed subacute intracerebral hemorrhages in the bilateral occipital lobes, which were seen as high signal intensities on T1-weighted imaging and as a dark rim on T2-weighted imaging. In the deep and subcortical white matter of the bilateral occipital lobes, cerebral edema was also seen as high signal intensities on T2-weighted (B) and fluid-attenuated inversion recovery imaging (C). MRI: magnetic resonance imaging; PRES: posterior reversible encephalopathy syndrome.
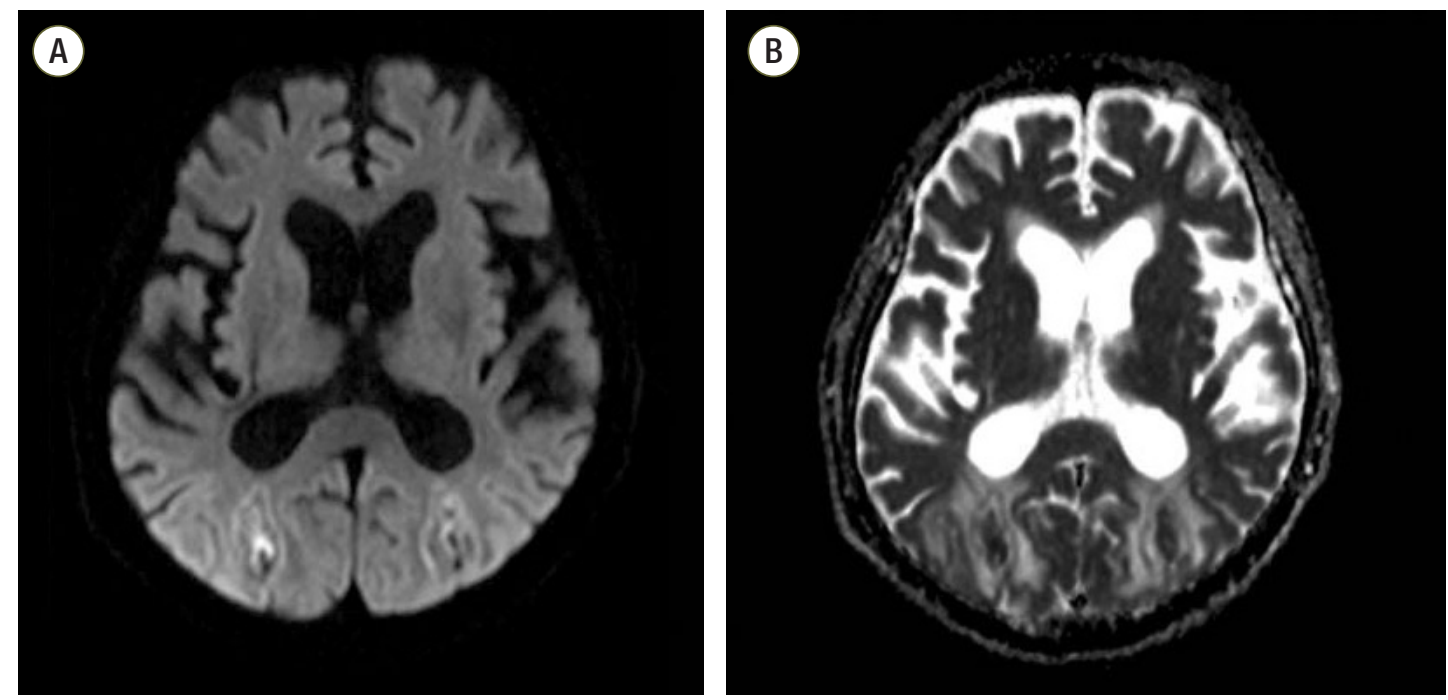

Fig. 2. MRI assessment of chronic change related to PRES with internal intracerebral hemorrhage. DWI (A) and ADC map imaging (B) did not reveal significant signal changes in the corresponding areas, except in the hemorrhagic foci. MRI: magnetic resonance imaging; PRES: posterior reversible encephalopathy syndrome; DWI: diffusion weighted imaging; ADC: apparent diffusion coefficient.

the patient woke up on postoperative day seven, he was able to obey simple commands without significant sign of neurologic deficit. Therefore, neurologic imaging studies were not performed. On postoperative day 14, tracheostomy was performed, and the patient was weaned off of the mechanical ventilator on postoperative day 17. Continuous renal replacement therapy was continued for 13 days postopera- tively and was discontinued after nine conventional hemodialysis sessions.

After recovering consciousness, the patient persistently complained of drowsiness, lethargy, and blurred vision in the general ward. A neurologic exam was conducted by a neurologist and the patient showed mild drowsy mental status with disorientation to time and place. His pupils 
were both isocoric and reactive to light stimuli. Magnetic resonance imaging (MRI) of the brain as well as electroencephalography were obtained on postoperative day 27 . Axial brain T1- and T2-weighted MRI disclosed subacute intracerebral hemorrhages (ICH) in the bilateral occipital lobes, and which were seen as high signal intensities on T1weighted images and with dark rim on T2-weighted images. In the deep and subcortical white matter of the bilateral occipital lobes, cerebral edema was also seen as high-signal intensities on T2-weighted and fluid attenuated inversion recovery (FLAIR) images (Fig. 1). Diffusion weighted image (DWI) and apparent diffusion coefficiency (ADC) map image did not show significant signal change in corresponding area, except the hemorrhagic foci. (Fig. 2) These findings were assessed as chronic change of PRES with internal intracerebral hemorrhage. Ophthalmologic examination was requested and the finding was almost within the normal range in a slit-lamp examination, funduscopy, and intraocular pressure measurement, except for significantly decreased visual acuity of unknown onset.

Although the patient was diagnosed with PRES, conservative management was maintained as there were no correctable medical conditions, such as uncontrolled hypertension, immunosuppressant or cytotoxic drug, infection, renal insufficiency, etc. On postoperative day 58, the patient was transferred to the Rehabilitation Medicine Department for comprehensive rehabilitation due to his remaining cognitive dysfunction. After one month, he was discharged from the hospital and remained well at home. According to the report of the outpatient clinic, he has still mild cognitive dysfunction and decreased visual acuity, although he is able to carry out the activities of daily living with minimal assistance.

\section{Discussion}

In 1996, Hinchey et al[1] first described a reversible posterior leukoencephalopathy syndrome in their report of 15 patients who had a reversible syndrome consisting of headache, altered mental functioning, seizures, and loss of vision associated with findings indicating predominantly posterior leukoencephalopathy, as seen on imaging studies obtained from 1998 through 1994. However, this term was replaced by PRES, as the involvement of gray matter was shown by
Casey et al[6] who discovered the utility of FLAIR MRI to detect distinguishing the cortical and subcortical edema of PRES.

The most common clinical symptoms and signs are seizures, altered mental status ranging from somnolence to stupor, headache, and visual disturbance such as hemianopia, visual neglect, and cortical blindness. There can also be nausea, vomiting, the Babinski sign, paresis, aphasia, and brainstem sign, depending on the type of brain lesion.[1,2,4,5,7-9]

There are various clinical conditions associated with the risk of PRES. The common triggering factors are abrupt arterial hypertension, eclampsia/pre-eclampsia, impaired renal function, an immunosuppressive agent such as cyclosporine or tacrolimus, a chemotherapy agent such as cytarabine, cisplatin or gemcitagine, allogenic bone-marrow transplantation, solid organ transplantation, infection/sepsis/shock, and autoimmune diseases such as systemic lupus erythematosus, Wegener's granulomatosis, systemic sclerosis, and polyarteritis nodosa.[2-5] In this present case, there were several possible conditions related to the risk of PRES, such as chronic kidney disease, septic shock and abrupt arterial hypertension during the operation. The presentations of PRES among the patients with chronic kidney disease or septic shock in the ICU are uncommon. But abrupt arterial hypertension, especially immediately after CPR like this patient, is very rare condition. And the ICH findings which were observed in the typical MRI lesions of PRES were considered to suggest arterial hypertension. Therefore, abrupt arterial hypertension is determined as a major risk factor for PRES in this case.

The pathophysiology of PRES is unknown. Bartynski recently summarized two, opposing theories explaining the development of the vasogenic edema of PRES. The more widely accepted theory is that abrupt severe hypertension exceeds the upper limit of cerebral auto-regulation, thus resulting in blood-brain barrier disruption with vasogenic edema. The other theory is that vasoconstriction caused by hypertension and auto-regulatory compensation results in brain hypoperfusion and ischemia, and finally in vasogenic edema.[3] In a recent study the Mayo clinic analyzed 113 patients with PRES, and Fugate et al. suggested that endothelial dysfunction might have a key role in the mechanism of normotensive PRES due to the high prevalence of autoimmune disease in PRES patients.[8] 
The prompt diagnosis of PRES is very important in order to begin appropriate patient management to prevent irreversible brain damage. Clinical symptoms and signs of PRES are vague, but the combination of these neurologic symptoms, especially for patients having predisposing factors, is crucial for further imaging evaluation of possible brain lesions. As a CT scan could show a low-attenuation lesion in the posterior cerebral hemisphere in only approximately $50 \%$ of PRES, MRI is the favored imaging modality for diagnosing PRES.[5] Due to the property of vasogenic cerebral edema, T2-weighted and FLAIR images show diffuse high-signal intensity. Normal or decreased signal intensity is seen in DWI images without reduction of the ADC value, and which accounts for the difference between the vasogenic edema of PRES and the cytotoxic edema of cerebral infarction.[10] As the term, PRES, signfies, vasogenic edema lesions are typically located bilaterally in the cortical gray matter and subcortical white matter of the parieto-occipital area. Several studies have indicated the simultaneous involvement of an atypical location. For example, the location might include the non-posterior cerebral hemisphere, cerebellum, brainstem, basal ganglia, deep white matter, and splenium with various frequency.[1,4,6,11,12]

Treatment of PRES is based on the elimination of possible triggering factors as well as the symptom management. Any causative medications should be withdrawn or reduced in dose.[1] Delivery or cesarean section would be the treatment of choice in preeclampsia/eclampsia patients. $[5,13]$ It is also important to vigorously control any infection, septic condition or autoimmune disease. Lowering blood pressure is essential for patients with hypertensive PRES. Blood pressure should be continuously monitored in the intensive care unit via an arterial line, and an intravenously administered, antihypertensive drug, such as sodium nitroprusside, labetalol or calcium channel blockers, is usually preferred. Angiotensin-converting enzyme inhibitors should be used cautiously in patients who are hypovolemic or in those with underlying renal artery stenosis due to potentially abrupt drops in blood pressure. The mean arterial pressure should be lowered by approximately $20 \%$ or to a diastolic blood pressure of $100 \mathrm{mmHg}$ during minutes to hours. Overaggressive reduction of blood pressure should be avoided as it could lead to end organ dysfunction and cerebral infarction.[14] Seizures should be actively treated using an anticonvulsant drug.[5,13] although long-term medication use is not required as seizures do not usually progress to chronic epilepsy.[7,9]

The neurologic symptoms of PRES usually are reversible. Hinchey et al.[1] reported that neurologic deficits resolved within two weeks in all of the patients they evaluated, and Roth et al.[9] reported that symptoms in their patients resolved on average after 7.5days. MRI abnormality is also reversible, although it takes longer than clinically demonstrated symptom recovery.[9] Although delayed diagnosis and treatment could result in irreversible brain damage, occasionally, despite appropriate treatment, poor neurologic outcomes have been reported.[6,11,14] Risk factors for irreversible brain damage are unknown, however, they might be associated with multimorbidity (sepsis, dementia) or intracerebral hemorrhage.[5,9]

Currently, PRES is a disease that is well-known to neurologists and neuroradiologists, although it remains unfamiliar to many physicians. As delayed diagnosis and treatment may lead to a poor neurologic prognosis with irreversible brain damage, physicians and intensivists should be aware of this syndrome. In particular, it is very difficult to detect a neurologic deficit in patients in an intensive care unit as critically ill patients are usually sedated and intubated. As in our patient, it is possible that PRES can develop even in patients in a critically ill condition. Therefore, intensivists should consider PRES if a patient with triggering factors does not experience mental status improvement even after the cessation of sedative drugs.

\section{ORCID}

Min Ae Keum http://orcid.org/0000-0002-1970-7327

Suk-Kyung Hong http://orcid.org/0000-0001-5698-0122

\section{References}

1) Hinchey J, Chaves C, Appignani B, Breen J, Pao L, Wang A, et al: A reversible posterior leukoencephalopathy syndrome. N Engl J Med 1996; 334: 494-500.

2) Bartynski WS: Posterior reversible encephalopathy syndrome, part 1: fundamental imaging and clinical features. AJNR Am J Neuroradiol 2008; 29: 1036-42. 
3) Bartynski WS: Posterior reversible encephalopathy syndrome, part 2: controversies surrounding pathophysiology of vasogenic edema. AJNR Am J Neuroradiol 2008; 29: 1043-9.

4) Bartynski WS, Boardman JF: Distinct imaging patterns and lesion distribution in posterior reversible encephalopathy syndrome. AJNR Am J Neuroradiol 2007; 28: 1320-7.

5) Roth C, Ferbert A: The posterior reversible encephalopathy syndrome: what's certain, what's new? Pract Neurol 2011; 11: 136-44.

6) Casey SO, Sampaio RC, Michel E, Truwit CL: Posterior reversible encephalopathy syndrome: utility of fluid-attenuated inversion recovery MR imaging in the detection of cortical and subcortical lesions. AJNR Am J Neuroradiol 2000; 21: 1199-206.

7) Lee VH, Wijdicks EF, Manno EM, Rabinstein AA: Clinical spectrum of reversible posterior leukoencephalopathy syndrome. Arch Neurol 2008; 65: 205-10.

8) Fugate JE, Claassen DO, Cloft HJ, Kallmes DF, Kozak OS, Rabinstein AA: Posterior reversible encephalopathy syndrome: associated clinical and radiologic find- ings. Mayo Clin Proc 2010; 85: 427-32.

9) Roth C, Ferbert A: Posterior reversible encephalopathy syndrome: long-term follow-up. J Neurol Neurosurg Psychiatry 2010; 81: 773-7.

10) Stott VL, Hurrell MA, Anderson TJ: Reversible posterior leukoencephalopathy syndrome: a misnomer reviewed. Intern Med J 2005; 35: 83-90.

11) Kwon S, Koo J, Lee S: Clinical spectrum of reversible posterior leukoencephalopathy syndrome. Pediatr Neurol 2001; 24: 361-4.

12) Ahn KJ, You WJ, Jeong SL, Lee JW, Kim BS, Lee JH, et al: Atypical manifestations of reversible posterior leukoencephalopathy syndrome: findings on diffusion imaging and ADC mapping. Neuroradiology 2004; 46: 978-83.

13) Servillo G, Bifulco F, De Robertis E, Piazza O, Striano P, Tortora F, et al: Posterior reversible encephalopathy syndrome in intensive care medicine. Intensive Care Med 2007; 33: 230-6.

14) Vaughan CJ, Delanty N: Hypertensive emergencies. Lancet 2000; 356: 411-7. 